\title{
Bichromatic state-insensitive trapping of caesium atoms
}

\section{M.M. Metbulut \& F. Renzoni}

To cite this article: M.M. Metbulut \& F. Renzoni (2015) Bichromatic state-insensitive trapping of caesium atoms, Journal of Modern Optics, 62:sup2, S52-S58, DOI:

10.1080/09500340.2015.1073807

To link to this article: http://dx.doi.org/10.1080/09500340.2015.1073807

\section{(c) 2015 The Author(s). Published by Taylor \& Francis}

曲 Published online: 12 Aug 2015.

Submit your article to this journal $\pi$

!ll Article views: 162

Q View related articles ¿

View Crossmark data 


\title{
Bichromatic state-insensitive trapping of caesium atoms
}

\author{
M.M. Metbulut* (D) and F. Renzoni \\ Department of Physics and Astronomy, University College London, London, UK
}

(Received 30 March 2015; accepted 3 July 2015)

\begin{abstract}
State-insensitive dipole trapping of multilevel atoms can be achieved by an appropriate choice of the wavelength of the trapping laser, so that the interaction with the different transitions results in equal AC Stark shifts for the ground and excited states of interest. However, this approach is severely limited by the availability of coherent sources at the required wavelength and of appropriate power. This work investigates state-insensitive trapping of caesium atoms for which the required wavelength of $935.6 \mathrm{~nm}$ is inconvenient in terms of experimental realization. Bichromatic state-insensitive trapping is proposed to overcome the lack of suitable laser sources. We first consider pairs of laser wavelengths in the ratio $1: 2$ and 1:3, as obtained via second- and third-harmonic generation. We found that the wavelength combinations 931.8$1863.6 \mathrm{~nm}$ and $927.5-2782.5 \mathrm{~nm}$ are suitable for state-insensitive trapping of caesium atoms. In addition, we examine bichromatic state-insensitive trapping produced by pairs of laser wavelengths corresponding to currently available highpower lasers. These wavelength pairs were found to be in the range of 585-588 $\mathrm{nm}$ and 623-629 for one laser and $1064-1080 \mathrm{~nm}$ for the other.
\end{abstract}

Keywords: magic wavelength; optical dipole trap; state-insensitive dipole trap; bichromatic trap; caesium atom; light shift

\section{Introduction}

Dipole trapping is of great importance for cold atom research. The ability to manipulate trapped neutral atoms plays an important role in many experiments in precision spectroscopy [1], optical clocks [2-4], quantum control [5] and quantum information processing [6]. Dipole trapping is produced by a spatial intensity gradient of the trapping laser field(s), which leads to a trapping potential for the atomic ground state. In all the experiments involving the interrogation of an optical transition, as for example in precision spectroscopy, the effect of the trapping light on the transition has to be taken into account. In general, the AC Stark shifts to the ground and excited states of the optical transition of interest are different, thus the trapping light leads to the formation of a differential shift between the two states. Such a differential AC Stark shift, which is positiondependent via the position-dependence of the laser intensity, has a number of drawbacks. It causes decoherence, thus limiting the applicability of dipole trapping in experiments requiring long coherence times [7]. It makes it difficult to perform precision spectroscopy on trapped atoms, as it results in a line shift and its position-dependence causes a line broadening. This also complicates the continuous monitoring of trapped atoms. Moreover, free-space cooling mechanisms cannot operate on trapped atoms due to the differential AC Stark shift. This also introduces difficulties in efficient loading of atoms into dipole traps.

In 1999, in the context of optical lattice clocks, Katori et al. [8] introduced a technique to keep the internal state of a trapped atom unaffected by the trapping light. By taking advantage of the multilevel structure of atoms, it was proposed to tune the trapping laser to a specific wavelength the so called magic wavelength - at which the differential light shift between the excited and the ground states of a particular transition vanishes. This is the result of the interaction of the dipole trap laser field with all the different transitions of the multilevel atom, with relative weights determined by the laser detuning. The effect was demonstrated for a strontium optical lattice clock, with state-insensitive trapping allowing for simultaneous laser cooling and dipole trapping. Later in 2003, state-insensitive trapping of caesium atoms confined in an optical cavity field at the magic wavelength of $935.6 \mathrm{~nm}$ was demonstrated by McKeever et al. [9] in the context of cavity quantum electrodynamics. It was shown that continuous monitoring of trapped atoms becomes possible with state-insensitive trapping. Later in 2010, state-insensitive trapping of caesium atoms in free space was also demonstrated [10]. However, in this case the size and depth of the trap is severely limited by the power of available laser sources at the required wavelength.

\footnotetext{
*Corresponding author. Email: ucapmmm@ucl.ac.uk
} 
The magic wavelengths for several other neutral atoms, such as $\mathrm{Mg}$ [11], $\mathrm{Hg}$ [12], $\mathrm{Rb}$ [13], $\mathrm{Ba}$ [14], $\mathrm{Ra}$ [14], Ca [15] and $\mathrm{Li}$ [16] have been determined. Magic wavelengths for Ryberg atoms have also been calculated $[17,18]$. However, in terms of experimental realizations of state-insensitive trapping, some of the identified magic wavelengths are of limited practical use. More specifically, the calculated magic wavelengths may be in an inappropriate frequency range for current laser technology, either in terms of wavelength or power required. In the present work, bichromatic trapping is investigated to overcome the above-listed problems associated with monochromatic state-insensitive trapping. Bichromatic trapping was first proposed by Arora et al. for rubidium atoms [19], as the magic wavelength for monochromatic trapping was inconvenient due to the atomic polarizability being too small for efficient trapping. In contrast to rubidium, the major obstacle for stateinsensitive monochromatic trapping of caesium atoms in free space is the lack of laser sources with sufficiently high power. Motivated by this, we investigate bichromatic stateinsensitive trapping of caesium atoms to determine combinations of magic wavelengths more convenient than the monochromatic magic wavelength of $935.6 \mathrm{~nm}$.

Bichromatic state-insensitive trapping relies on the combined action of two independent laser fields with different wavelengths. One of the lasers is used as the trap laser and the other one is used as a control laser to compensate for the differential light shift between the ground and excited states of the atomic transition of interest. Bichromatic stateinsensitive trapping is more flexible that its monochromatic counterpart, as several different combinations of parameters of the two lasers may lead to state-independent trapping. Thus, bichromatic trapping may be useful to extend the range of possible wavelengths that can be used for stateinsensitive trapping.

This paper is organized as follows. First, the case of laser wavelengths in the $1: 2$ and 1:3 ratio is considered, with particular attention to the infrared region of spectrum. This corresponds to pairs of laser fields with one obtained from the other one via second- or third-harmonic generation. Next, wavelength combinations attainable by currently available high-power lasers were considered, and appropriate combinations for state-insensitive trapping determined. Finally, the effect of unwanted laser intensity and wavelength variations is discussed.

\section{Numerical results}

We investigate bichromatic schemes for caesium atoms, which provide state-insensitive trapping with respect to the $\mathrm{D}_{2}$ line transition, i.e. with no differential light shift for that transition.

Our numerical approach follows standard procedure. We evaluate separately the AC Stark shift of the ground state $6 S_{1 / 2}(F=4)$ and the excited state $6 P_{3 / 2}(F=5)$ in the presence of both trap and control lasers as functions of the laser fields' wavelengths. This is done using timeindependent perturbation theory. Then we determine the crossing point of the AC Stark shifts for the two states. This gives the wavelength combination required for stateindependent trapping. All the atomic hyperfine levels and their corresponding Zeeman sublevels are included in our calculation. In this way, we can identify the required combination of magic wavelengths for situations in which the trapped atoms are prepared in specific Zeeman sublevels.

Consider an atom driven by a laser field with intensity $I_{L}$ and frequency $\omega_{0}$. The AC Stark shift experienced by the atomic level $|i\rangle=\left|F_{i} m_{i}\right\rangle$ as a result of the laser coupling to a set of states $|j\rangle=\left|F_{j} m_{j}\right\rangle$ is given by

$$
\begin{aligned}
\Delta E_{i}= & \left(2 F_{i}+1\right) I_{L} \sum_{j} \frac{3 \pi c^{2} A_{i j}}{2 \Delta \omega_{0}^{3}}\left(2 F_{j}+1\right)\left(2 J_{j}+1\right) \\
& \times\left(\begin{array}{ccc}
F_{j} & 1 & F_{i} \\
m_{j} & q & -m_{i}
\end{array}\right)^{2}\left\{\begin{array}{ccc}
J_{i} & J_{j} & 1 \\
F_{j} & F_{i} & I
\end{array}\right\}^{2}
\end{aligned}
$$

where $A_{i j}$ is the Einstein A-coefficient for the transition $|i\rangle \leftrightarrow|j\rangle, I$ the nuclear spin and $\Delta$ the laser field detuning from resonance with the considered transition. The parameter $q$ parametrizes the polarization of the light: $q= \pm 1$ for $\sigma^{ \pm}$light and $q=0$ for linearly polarized light.

The AC Stark shift produced by the trap laser $\left(\Delta E_{i}^{t}\right)$ and control laser $\left(\Delta E_{i}^{c}\right)$ are calculated separately via Equation 1 , with the total AC Stark shift given by their sum.

All calculations presented in this work are carried out for linear polarization $(q=0)$ of the trap and control laser fields. This is the most widely used configuration for dipole trapping, as the ground state Zeeman sublevels remain degenerate in the presence of the trapping light. Calculations of the AC Stark shift of the $|i\rangle$ states $6 S_{1 / 2}$ $(F=4)$ and $6 P_{3 / 2}(F=5)$ are performed, including contributions from the coupling with the $|j\rangle$ states up to $15 \mathrm{~S}, 11 \mathrm{P}$ and $11 \mathrm{D}$.

\subsection{Magic wavelength pairs for $\lambda_{c}=2 \lambda_{t}$ and $\lambda_{c}=3 \lambda_{t}$}

We consider here bichromatic trapping schemes with the trap and control laser wavelengths in the ratio $2: 1$ and $3: 1$, as obtained from a single laser source via second- or thirdharmonic generation, respectively. The calculations were first performed for equal intensity of $2 \times 10^{9} \mathrm{~W} / \mathrm{m}^{2}$ of the trap and the control lasers. To determine the wavelengths leading to state-independent trapping, the AC Stark shifts of the ground and the excited state of the $F_{g}=4 \rightarrow F_{e}=5 \mathrm{D}_{2}$ line transition were evaluated as functions of the trap laser wavelength for the two configurations of interest: $\lambda_{\text {control }}=$ $2 \lambda_{\text {trap }}$ and $\lambda_{\mathrm{c}}=3 \lambda_{\mathrm{t}}$. Results of the calculations are shown in Figure 1. As expected, the excited state exhibits splittings, whereas the ground state remains degenerate. From the numerical results of Figure 1 we identify the following combinations of magic wavelengths of the trap and the 


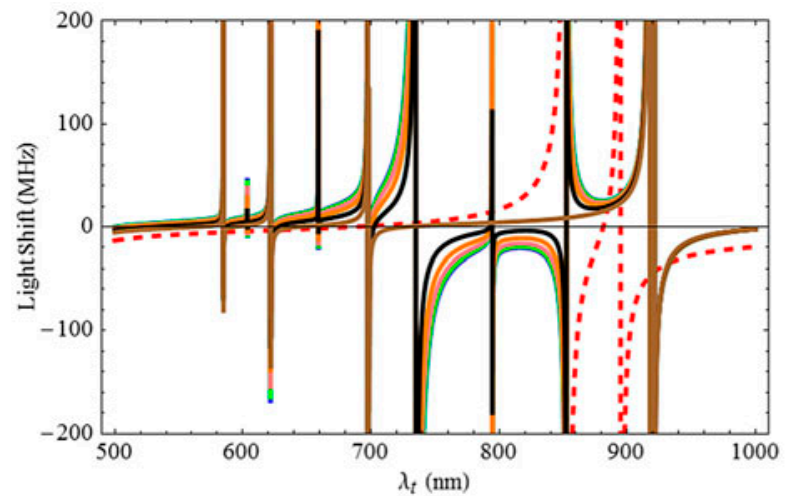

(a) $\lambda_{\text {control }}=2 \lambda_{\text {trap }}$.

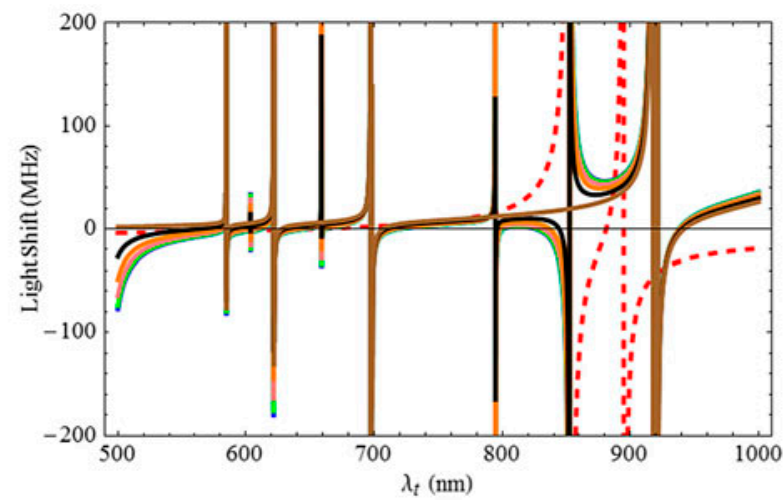

(b) $\lambda_{\text {control }}=3 \lambda_{\text {trap }}$.

Figure 1. AC Stark shift for the $F_{g}=4 \rightarrow F_{e}=5$ transition as function of trap laser wavelength for $(a) \lambda_{\mathrm{c}}=2 \lambda_{\mathrm{t}}$ and $(b) \lambda_{\mathrm{c}}=3 \lambda_{\mathrm{t}}$. The intensities of the trap and control laser is equal and kept at $2 \times 10^{9} \mathrm{~W} / \mathrm{m}^{2}$. Red dashed line is the ground state $6 S_{1 / 2}$, blue, green, pink, orange, black and brown lines are the excited state $6 P_{3 / 2}$ with $m=0, m= \pm 1, m= \pm 2, m= \pm 3, m= \pm 4$ and $m= \pm 5$ Zeeman sublevels, respectively. (The colour version of this figure is included in the online version of the journal.)

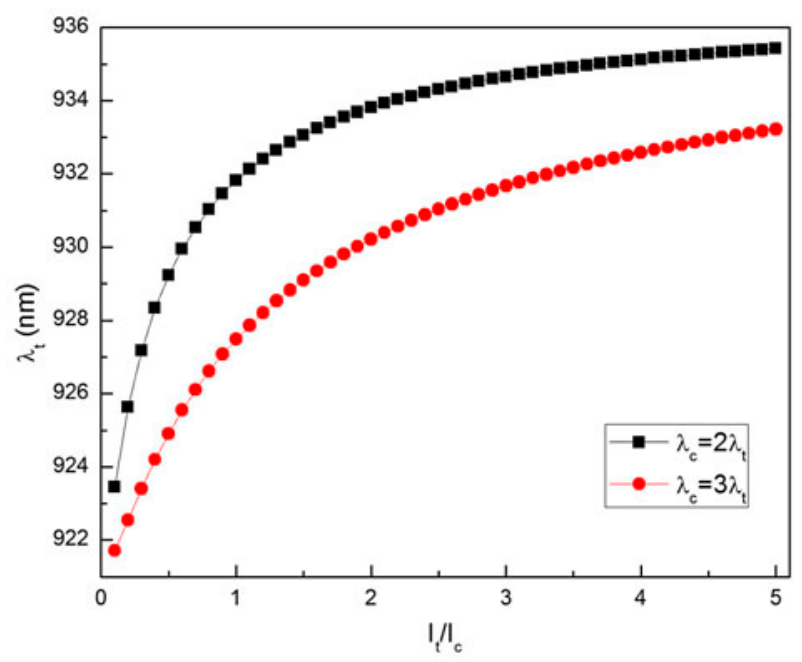

Figure 2. Trap laser wavelength required for state-independent trapping vs. the ratio of trap and control laser intensities. The two data-sets correspond to the cases $\lambda_{\text {control }}=2 \lambda_{\text {trap }}$ and $\lambda_{\text {control }}=$ $3 \lambda_{\text {trap. }}$ The lines are guides for the eyes. (The colour version of this figure is included in the online version of the journal.)

control lasers: $931.8-1863.6 \mathrm{~nm}$ for the case $\lambda_{\mathrm{c}}=2 \lambda_{\mathrm{t}}$ and $927.5-2782.5 \mathrm{~nm}$ for $\lambda_{\mathrm{c}}=3 \lambda_{\mathrm{t}}$, respectively.

Moreover, it is worth noting that, in the case of bichromatic trapping, the wavelength combination leading to state-independent trapping depends on the ratio of the trap and control laser intensities, thus introducing a degree of tunability of the magic wavelength. Such an the effect is illustrated in Figure 2, where the results of the calculation of the magic wavelength as a function of the intensity ratio of the trap and control lasers are reported for both $\lambda_{\mathrm{c}}=2 \lambda_{\mathrm{t}}$ and $\lambda_{c}=3 \lambda_{t}$. The data indeed demonstrate tunability of the magic wavelength in excess of $10 \mathrm{~nm}$, by changing the relative intensities of the lasers. Thus, bichromatic trapping significantly extends the range of possible combinations of magic wavelengths with respect to the monochromatic case.

\subsection{Magic wavelength pairs corresponding to available high-power lasers}

As previously pointed out, our main motivation for investigating bichromatic state-insensitive trapping of caesium atoms is the absence of a laser source at the magic wavelength of $935.6 \mathrm{~nm}$ with sufficiently high power for atom trapping in free space. We thus here investigate combinations of magic wavelength, which can be produced by highpower lasers which are currently available, in the sense of there being a suitable laser gain medium that provides high power at the required wavelength.

For all calculations presented in the following of the paper the beam waists of both trap and control lasers are equal to $10 \mu \mathrm{m}$. The possible control laser wavelengths appropriate to achieve state-insensitive trapping were found to be $1064,1070,1075$ and $1080 \mathrm{~nm}$ that can be achieved by Nd:YAG and ytterbium fibre lasers. The corresponding magic trap wavelengths accessible by high-power lasers for all these control laser wavelengths were found in the range between 585-588 and 623-629 nm, which can be obtained using orange and red Raman fibre lasers, in which a Yb-fibre laser generates stimulated Raman scattering in a second fibre, with the resulting infrared Raman emission frequency doubled into the orange/red region of the visible.

The calculations of the AC Stark shift of the $6 S_{1 / 2} F_{g}=$ $4 \rightarrow 6 P_{3 / 2} F_{e}=5$ transition for the control laser wavelength of $1064 \mathrm{~nm}$ is presented in Figure 3. Two magic trap wavelengths were identified: 586.1 and $627.8 \mathrm{~nm}$.

The calculations presented in the previous Section pointed out that, in the case of bichromatic trapping, it is 

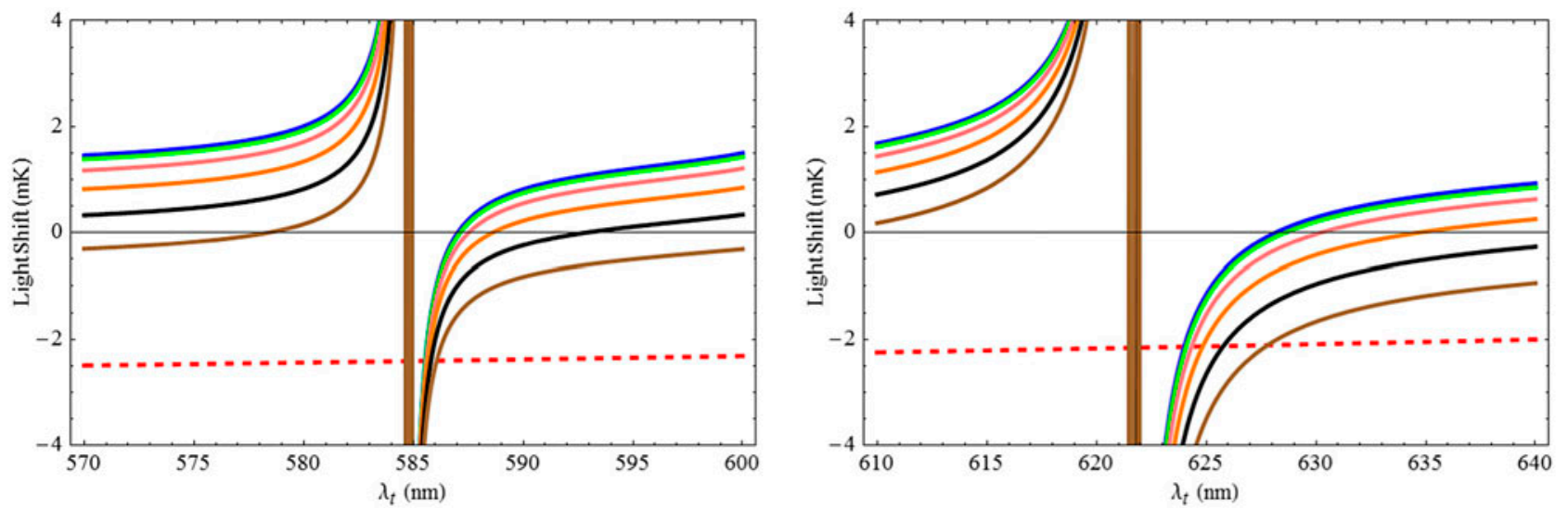

Figure 3. AC Stark shift of ground and excited states of the $6 S_{1 / 2} F_{g}=4 \rightarrow 6 P_{3 / 2} F_{e}=5$ transition as functions of the trap laser wavelength. The control laser wavelength is $1064 \mathrm{~nm}$ and the power of both trap and control beams is $2 \mathrm{~W}$. The red dashed line refers to the ground state $6 S_{1 / 2}$ and the other bold lines refer to the Zeeman sublevels of the excited state $6 P_{3 / 2}$, with the same colour code as in Figure 1. The left and right panel evidence two different regions of the examined wavelength range, with each of the regions containing a value of trap laser wavelength producing state-independent trapping. (The colour version of this figure is included in the online version of the journal.)

possible to tune the combination of magic wavelength of the trap and control lasers by varying their intensity ratio. Such tunability applies also to the combinations of laser wavelength considered in this section. The dependence of the required ratio between the trap and the control laser intensities on the wavelength of the trap laser is shown in Figure 4 for control laser wavelength of 1064, 1070, 1075 and $1080 \mathrm{~nm}$. For all of the four control laser wavelengths, the required intensity ratio of the trap and the control lasers exhibits an approximately linear dependence on the corresponding wavelength of the trap laser, thus offering precise tunability over a wide range of wavelengths. Nevertheless, there is a limit on the possible tuning range of the magic wavelength. Figure 4 shows that as the trap laser wavelength increases, the intensity of the trap laser relative to the control laser also has to increase to maintain the condition of state-independent trapping. However, the increase in trap laser detuning decreases the trap depth. This is quantified in Figure 5, which evidences that the magnitude of the trap depth is inversely proportional to the trap wavelength. This sets a limit to the range of trap wavelengths usable for stateindependent trapping.

An important aspect for practical applications is the lifting of the excited state degeneracy produced by the trapping light. As Figure 3 indicates, the excited state Zeeman sublevels are in general split, with the amount of splitting dependent on the trapping light wavelengths combination. Thus, each sublevel has a different combination of trap/control magic wavelength. This poses limitations on the use of state-independent traps in conjunctions with mechanisms, which may require efficient optical pumping. This is, for example, the case of optical molasses during the loading of a dipole trap from a magneto-optical trap. We thus investigated in detail the splitting between sublevels as a function of the experimental parameters, within the subset of these parameters which lead to state-independent trapping. We are in particular interested in determining the conditions for the splitting between the sublevels to be less than $-2 \Gamma$, with $\Gamma$ the atomic excited state linewidth, which is a typical detuning for optical molasses. As the trap depth increases, in fact, the sublevels split further. Thus, to maintain the splitting between the sublevels below $2 \Gamma$, the trap depth should be kept under a certain value. To determine such a value, the trap depth was evaluated as function of the magic trap wavelength for all control laser wavelengths for a fixed $2 \Gamma$ splitting between the sublevels, with results in Figure 6. It follows that, with the proposed laser combinations, the deepest trap achievable to be able to take advantage of simultaneous laser cooling and dipole trapping is $0.95 \mathrm{mK}$ for a trap laser wavelength of 585.3 $\mathrm{nm}$.

In addition, the dependence on the trap laser wavelength of the splitting between the Zeeman sublevels was investigated, with results in Figure 7. The actual value of the combination of magic wavelength affects the magnitude of the splitting between the Zeeman sublevels. The lowest splitting occurs at magic wavelength pairs of 586.2-1064 and $630-1064 \mathrm{~nm}$. As the data indicates, any deviation from these values leads to an increase in the magnitude of the splitting. Given the very different trap depth obtainable at these different wavelength, the magic wavelength pair $586.2-1064 \mathrm{~nm}$ is more favourable for state-independent trapping.

\subsection{Effects of laser intensity and wavelength variations}

In a bichromatic state-insensitive trap, variations in laser intensity or wavelength lead to a violation of the conditions 

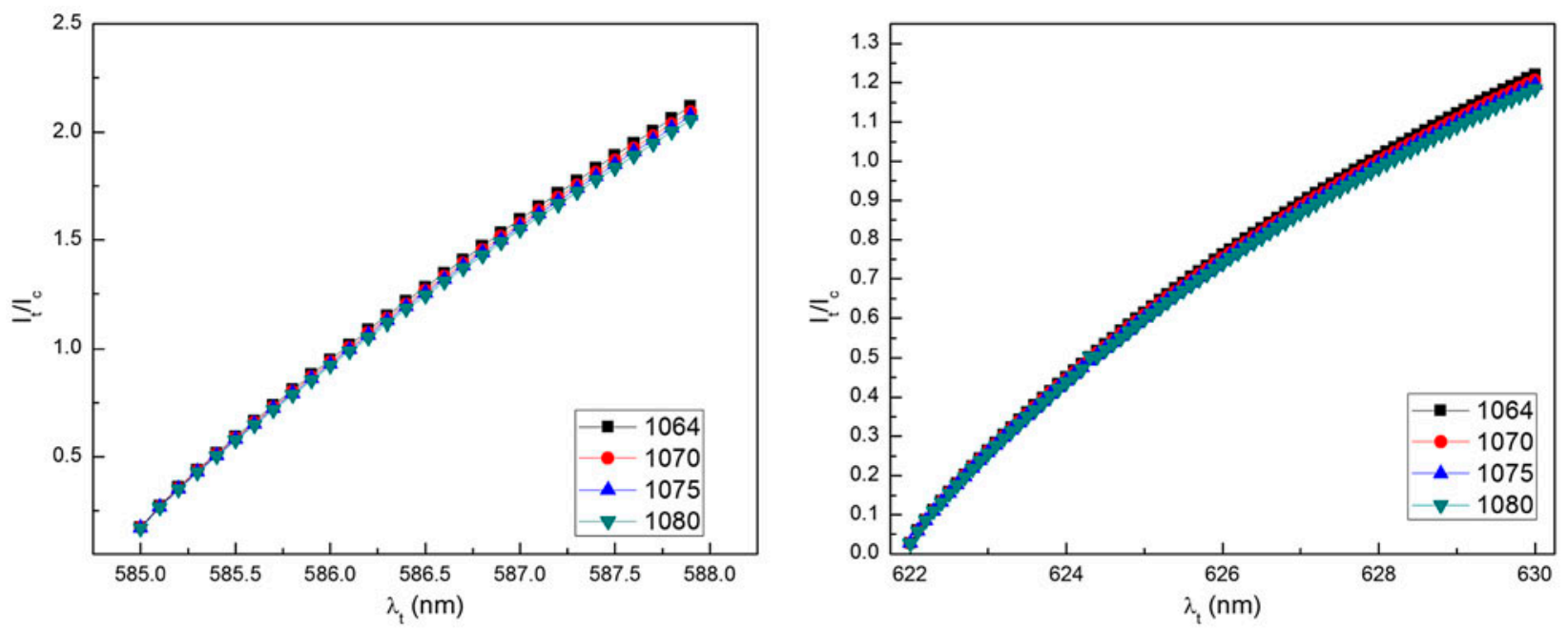

Figure 4. Dependence of the ratio between the trap and the control lasers intensities on the wavelength of the trap laser, as required to maintain state-independent trapping. Different data-sets correspond to different control laser wavelength of 1064, 1070, 1075 and 1080 $\mathrm{nm}$. The left panel refers to state-insensitive trapping obtained with a trap laser wavelength in the range of 585-587.9 $\mathrm{nm}$, the right panel refers to state-insensitive trapping obtained with a trap laser wavelength in the range of $623-630 \mathrm{~nm}$. (The colour version of this figure is included in the online version of the journal.)
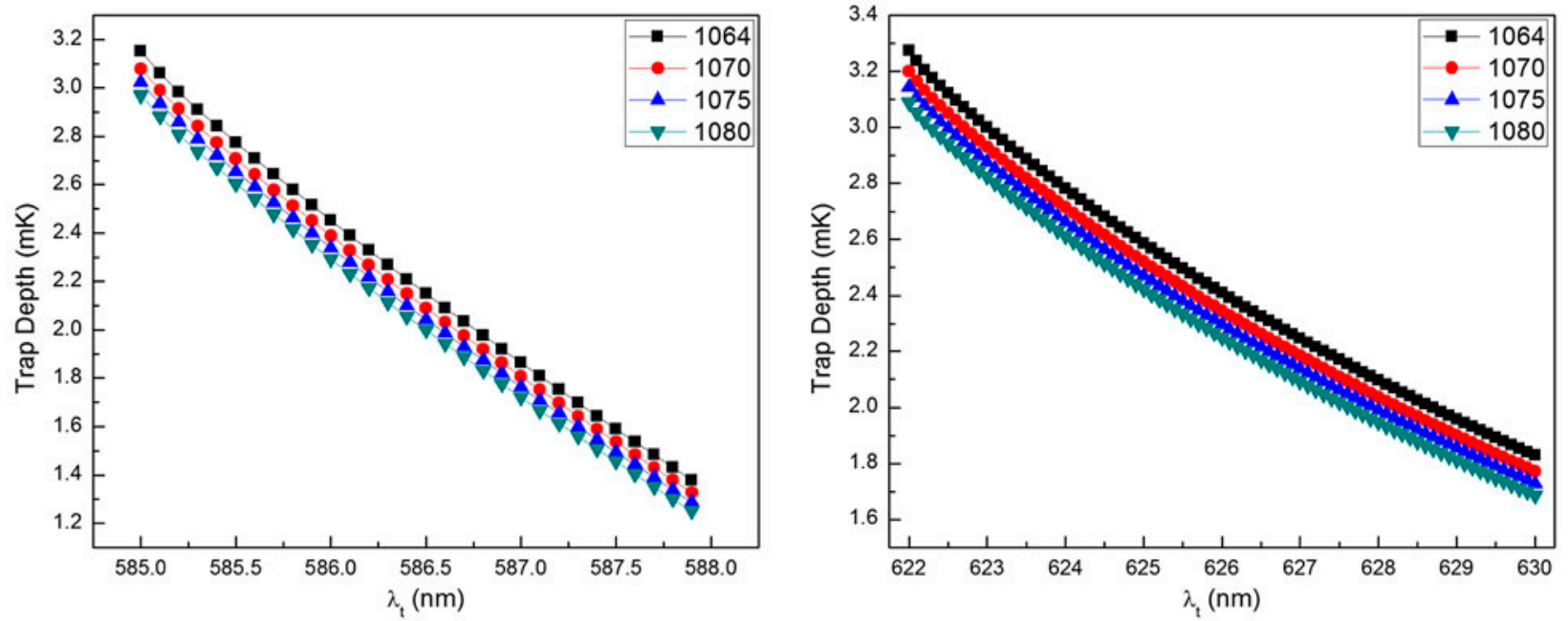

Figure 5. Variation of obtained trap depth with trapping laser wavelength. All data correspond to state-independent trapping, as obtained by appropriately tuning the ratio between the trap and the control lasers intensities. Different data-sets correspond to different control laser wavelength of 1064, 1070, 1075 and $1080 \mathrm{~nm}$. (The colour version of this figure is included in the online version of the journal.)

for state-insentive trapping. The effect of these variations is quantitatively investigated in this section. Specifically, we investigated the effect of intensity variations of the trap and control lasers on the AC Stark shift experienced by the ground and the excited states for the magic wavelength pair of 623.5-1064 $\mathrm{nm}$ and a trap depth of $1 \mathrm{mK}$. The differential AC Stark shift was evaluated for a variation of $\pm 15 \%$ in the intensity ratio of the trap and the control lasers. Different excited state Zeeman sublevels experience different shifts induced by the variations in laser intensity. Figure 8 shows that the different shifts of the different Zeeman sublevels of the excited state vary linearly with the intensity ratio. Also we notice that a variation in the intensity ratio of lasers causes a variation in the magic wavelength of each Zeeman sublevel. As the intensity ratio deviates from the value corresponding to state-independent trapping for a specific sublevel of the excited state, it approaches the required value of intensity ratio for the next sublevel. 


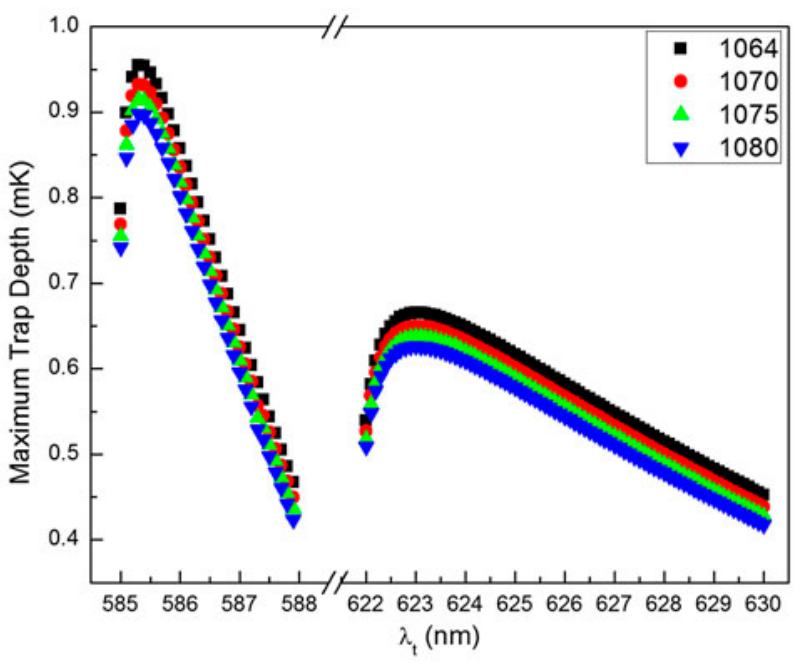

Figure 6. Trap depth vs. wavelength of the trap laser for a splitting between excited state sublevels equal to $2 \Gamma$. The condition of state-independent trapping is satisfied for all reported data. The power of the control laser used in the calculation is $0.968 \mathrm{~W}$. (The colour version of this figure is included in the online version of the journal.)

The other experimental parameter whose variations affect the condition of state-independent trapping is the wavelength of the two lasers. This effect was investigated by considering the variations of the laser wavelengths from the required value. The differential AC Stark shift between the ground and the excited states of the caesium atom was calculated for a variation of the trap laser wavelength up to a value $\Delta \lambda_{t}= \pm 1.5 \mathrm{~nm}$, while keeping the intensity ratio fixed for a combination of magic wavelengths of 623.5$1064 \mathrm{~nm}$. The differential light shift corresponding to the

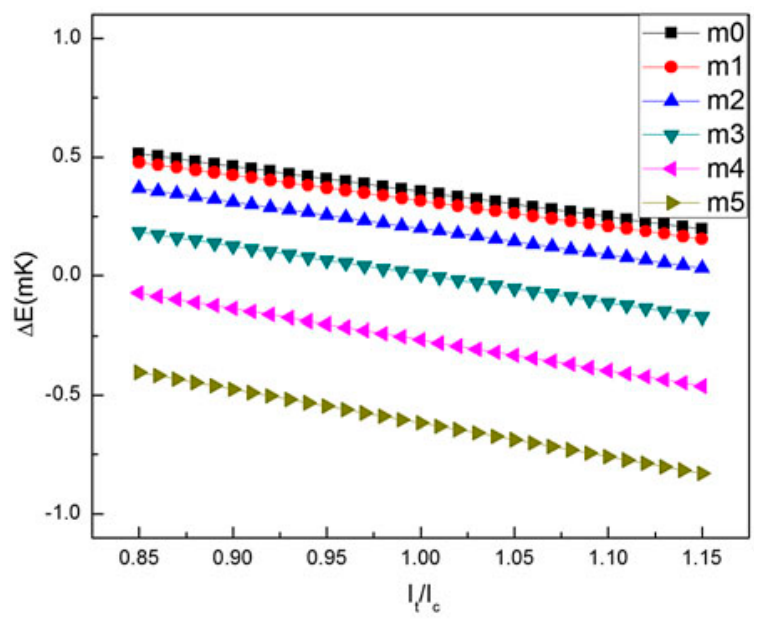

Figure 8. Differential AC Stark shift produced by laser intensity variations. Individual differential AC Stark shifts of the different excited state sublevels are reported. The power of the control laser used in the calculation is $2 \mathrm{~W}$. (The colour version of this figure is included in the online version of the journal.)

excited state sublevel, which experiences the smallest shift was calculated. Results of the calculations are reported in Figure 9, where the effects of the trap laser wavelength variations are quantified. We notice that as the trap laser wavelength approaches the $8 D_{3 / 2}$ transition at $621.7 \mathrm{~nm}$ the differential AC Stark shift increases significantly. Away from this transition, as the trap laser wavelength increases the differential AC Stark shift exhibit an oscillatory value around zero.
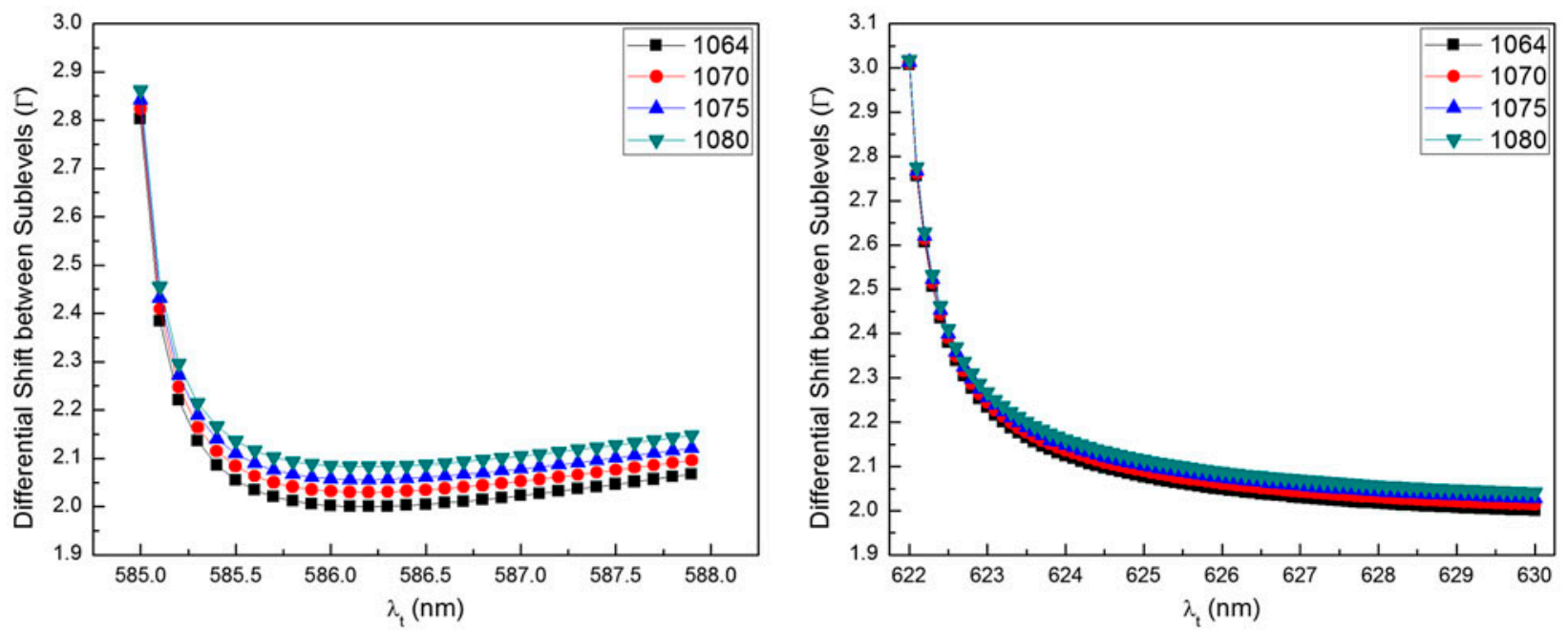

Figure 7. Dependence on the trap laser wavelength of the splittings between the Zeeman sublevels of the $6 P_{3 / 2}$ excites state for different control laser wavelengths of 1064, 1070, 1075 and $1080 \mathrm{~nm}$. The splitting is reported in units of the excited state linewidth. The power of the control laser used in the calculation is 0.7 and $0.495 \mathrm{~W}$, for the left and right panel, respectively. (The colour version of this figure is included in the online version of the journal.) 


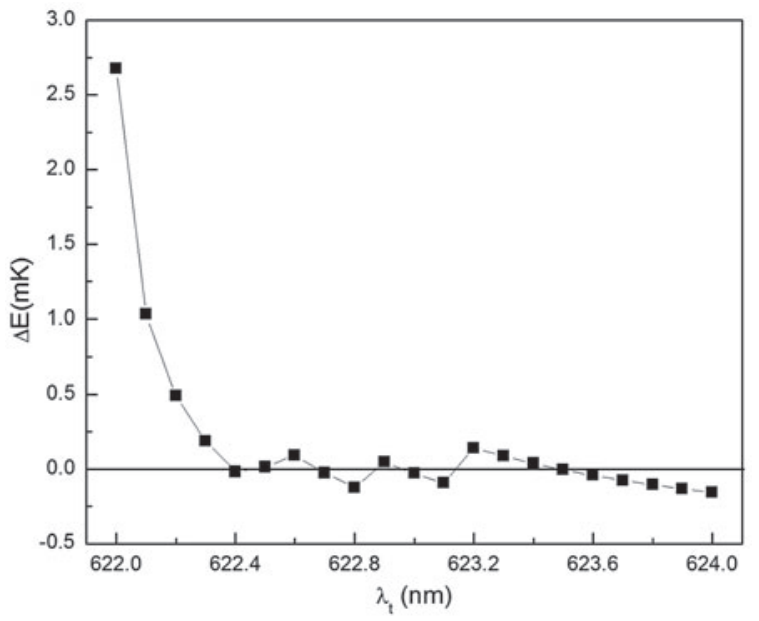

Figure 9. Differential AC Stark shift between excited and ground state of the caesium atom as a function of the trap laser wavelength. The differential light shift of the excited state sublevel which experiences the smallest shift is displayed. Calculations are for an intensity ratio $I_{t} / I_{c}=0.36053$. The power of the control laser used in the calculation is $2 \mathrm{~W}$.

\section{Conclusion}

In this work, bichromatic state-insensitive trapping of caesium atoms was investigated to overcome the problem of lack of sufficiently intense laser sources for free space monochromatic trapping. Our results identified several combinations of magic wavelengths promising for practical realizations. First, combinations of magic wavelengths were determined as 931.8-1863.6 and 927.5-2782.5 $\mathrm{nm}$ for the specific cases of $\lambda_{\mathrm{c}}=2 \lambda_{\mathrm{t}}$ and $\lambda_{\mathrm{c}}=3 \lambda_{\mathrm{t}}$ with equal power. The required laser beams may be derived from a single laser source via second- or third-harmonic generation. Then magic wavelength pairs achievable by two currently available high-power lasers were identified. We found that for a control laser wavelength of 1064, 1070, 1075 and 1080 $\mathrm{nm}$ the corresponding magic trap wavelength accessible by high-power lasers for all these control laser wavelengths were in the range between 585-588 nm and 623-629 nm. These wavelength combinations can be implemented by employing a red or orange Raman fibre laser as trap laser and a Nd:YAG or ytterbium fibre laser as control laser.

This work demonstrates that bichromatic trapping extends the range of possible magic wavelengths compared to the specific magic wavelength associated to a single laser scheme. Bichromatic trapping also introduces a degree of tunability of the magic wavelength, as by varying the trap/control laser intensity ratio it is possible to vary the wavelength combination leading to state-independent trapping. Therefore the limitations imposed on the power and wavelength of the laser source in the case of the single laser scheme is overcome by bichromatic trapping. In this perspective, bichromatic schemes for state-insensitive trapping are more advantageous than single laser schemes.

\section{Acknowledgment}

We thank Dr Luca Marmugi (UCL) for critical reading the manuscript.

\section{Funding}

This work was supported by EPSRC [grant number EP/H049231/1].

\section{ORCID}

M.M. Metbulut (D) http://orcid.org/0000-0002-6870-742x

\section{References}

[1] Ye, J.; Kimble, H.J.; Katori, H. Science 2008, 320, 1734-1738.

[2] Katori, H.; Hashiguchi, K.; Illinova, E.Y.; Ovsiannikov, V.D. Phys. Rev. Lett. 2009, 103, 153004.

[3] Takamoto, M.; Hong, F.L.; Higashi, R.; Katori, H. Nature 2005, 435 (7040), 321-324.

[4] Ludlow, A.D.; Zelevinsky, T.; Campbell, G.K.; Blatt, S.; Boyd, M. M.; De Miranda M.H.G.; Martin, M.J. Science 2008, 5871, $1805-1808$.

[5] Jones, M.P.A.; Beugnon, J.; Gaetan, A.; Zhang, J.; Messin, G.; Browaeys, A.; Grangier, P. Phys. Rev. A 2007, 75, 040301

[6] Dumke, R.; Volk, M.; Müther, T.; Buchkremer, F.B.J.; Birk1, G.; Ertmer, W. Phys. Rev. Lett. 2002, 89, 097903.

[7] Çetinbas, M. J. Phys. A: Math. Theor. 2009, 42, 145302.

[8] Katori, H.; Ido, T.; Kuwata-Gonokami, M. J. Phys. Soc. Jpn. 1999, 68, 2479-2482.

[9] McKeever, J.; Buck, J.R.; Boozer, A.D.; Kuzmich, A.; Nägerl, H.-C.; Stamper-Kurn, D.M.; Kimble, H.J. Phys. Rev. Lett. 2003, 90, 133602.

[10] Phoontong, P.; Douglas, P.; Wickenbrock, A.; Renzoni, F. Phys. Rev. A 2010, 82, 013406.

[11] Ovsiannikov, V.D.; Palchikov, V.G.; Taichenachev, A.V.; Yudin, V.I.; Katori, H.; Takamoto, M. Phys. Rev. A 2007, 75, 02051.

[12] Yi, L.; Mejri, S.; McFerran, J.J.; Le Coq, Y.; Bize, S. Phys. Rev. Lett. 2010, 107, 073005.

[13] Lundblad, N.; Schlosser, M.; Porto, J.V.; Yudin, V.I.; Katori, H.; Takamoto, M. Phys. Rev. A 2007, 81, 031611.

[14] Dammalapati, U.; Santra, B.; Willmann, L. J. Phys. B: At. Mol. Opt. Phys. 2012, 45, 025001.

[15] Degenhardt, C.; Stoehr, H.; Sterr, U.; Riehle, F.; Lisdat, C. Phys. Rev. A 2004, 70, 023414.

[16] Safronova, M.S.; Safronova, U.I.; Clark, C.W. Phys. Rev. A 2012, 86, 042505 .

[17] Mukherjee, R.; Millen, J.; Nath, R.; Jones, M.P.A.; Pohl, T. J. Phys. B: At. Mol. Opt. Phys. 2011, 44, 184010

[18] Goldschmidt, E.A.; Norris, D.G.; Koller, S.B.; Wyllie, R.; Brown, R.C.; Porto, J.V. arXiv:1503.02881.

[19] Arora, B.; Safronova, M.S.; Charles, W.C. Phys. Rev. A 2010 , 82,022509 . 